\title{
Toward a possible next geomagnetic transition?
}

\author{
A. De Santis ${ }^{1,2}$, E. Qamili ${ }^{1}$, and L. Wu ${ }^{3,4}$ \\ ${ }^{1}$ Istituto Nazionale di Geofisica e Vulcanologia, Sezione Roma 2, Roma, Italy \\ 2Università “G. D’Annunzio", Campus Universitario, Chieti, Italy \\ ${ }^{3}$ Northeastern University, Shenyang, China \\ ${ }^{4}$ China University of Mining and Technology, Xuzhou, China \\ Correspondence to: A. De Santis (angelo.desantis@ingv.it)
}

Received: 12 June 2013 - Published in Nat. Hazards Earth Syst. Sci. Discuss.: 27 September 2013

Revised: - - Accepted: 28 November 2013 - Published: 23 December 2013

\begin{abstract}
The geomagnetic field is subject to possible reversals or excursions of polarity during its temporal evolution. Considering that: (a) in the last 83 million yr the typical average time between one reversal and the next (the so-called chron) is around $400000 \mathrm{yr}$, (b) the last reversal occurred around $780000 \mathrm{yr}$ ago, (c) more excursions (rapid changes in polarity) can occur within the same chron and (d) the geomagnetic field dipole is currently decreasing, a possible imminent geomagnetic reversal or excursion would not be completely unexpected. In that case, such a phenomenon would represent one of the very few natural hazards that are really global. The South Atlantic Anomaly (SAA) is a great depression of the geomagnetic field strength at the Earth's surface, caused by a reverse magnetic flux in the terrestrial outer core. In analogy with critical point phenomena characterized by some cumulative quantity, we fit the surface extent of this anomaly over the last $400 \mathrm{yr}$ with power law or logarithmic functions in reverse time, also decorated by logperiodic oscillations, whose final singularity (a critical point $t_{\mathrm{c}}$ ) reveals a great change in the near future $(2034 \pm 3 \mathrm{yr})$, when the SAA area reaches almost a hemisphere. An interesting aspect that has recently been found is the possible direct connection between the SAA and the global mean sea level (GSL). That the GSL is somehow connected with SAA is also confirmed by the similar result when an analogous critical-like fit is performed over GSL: the corresponding critical point $(2033 \pm 11 \mathrm{yr})$ agrees, within the estimated errors, with the value found for the SAA. From this result, we point out the intriguing conjecture that $t_{\mathrm{c}}$ would be the time of no return, after which the geomagnetic field could fall into an irreversible process of a global geomagnetic transition that could be a reversal or excursion of polarity.
\end{abstract}

\section{Introduction}

The magnetic field of the Earth changes in time and space, in an irregular fashion, including dramatic manifestations such as the geomagnetic reversals or excursions, when the magnetic polarities exchange in sign, so that the geomagnetic south becomes north and vice versa (e.g., Jacobs, 1994). Over the last 83 million years we count 184 reversals (Cande and Kent, 1995). From the facts that: (a) the typical average time between a reversal and another (the so-called chron) is around $400000 \mathrm{yr}$, (b) the last reversal occurred around $780000 \mathrm{yr}$ ago, (c) more excursions (rapid changes in polarity) can occur within the same chron and (d) the geomagnetic field dipole is currently decreasing, a possible imminent geomagnetic reversal or excursion would not be completely unexpected. Such a phenomenon would represent one of the very few natural hazards that are really global, because it would affect the whole globe, although the detailed consequences over the planet, in general, and the biosphere, in particular, are not completely known. For instance, we recall a presumed link with mass extinctions (Raup, 1985; Courtillot and Besse, 1997; but see also Constable and Korte, 2006).

In the last $25 \mathrm{yr}$, some papers have appeared suggesting that an imminent reversal could occur (e.g., De Santis et al., 2004 and the references therein). The recent dipole decrease is considered part of a trend that has continued for the last $2000 \mathrm{yr}$ (Merrill and McElhinny, 1983) and a more rapid poleward drift of the dipole axis in the past $50 \mathrm{yr}$ has also been suggested (Amit et al., 2010). Analyzing the past $150 \mathrm{yr}$ of magnetic data, a more significant decay of the geomagnetic dipole intensity was found (Gubbins, 1987; Gubbins et al., 2006), much faster than the rate of free decay 
in the Earth's core (Olson and Amit, 2006). Most of this decay stems from the Southern Hemisphere, as shown by Gubbins (1987), who also suggested a direct correlation between the dipole decrease and the westward movements of a pair of reverse fluxes under South Africa. Other studies (e.g., Hulot et al., 2002; Constable, 2011) confirm the presence, at the core mantle boundary (CMB), of two reverse flux features: in particular, one is placed inside the tangent cylinder near the North Pole and the other is a large reverse flux patch under the Southern Atlantic that has been associated with the rapid decay of the field strength. Other authors have concentrated their studies on understanding the mechanism of magnetic polar reversals in dynamo numerical models (e.g., Glatzmaier and Roberts, 1995). Flux patches of reversed polarity appear at low or mid latitude prior to a reversal and then migrate polewards, thus reducing the axial dipole component (Wicht and Olson, 2004; Takahashi et al., 2005; Aubert et al., 2008; Wicht et al., 2009; Wicht and Christensen, 2010; Christensen, 2011). All these results are in agreement with early stages of a dipole collapse in the numerical dynamo model by Olson et al. (2009). In a detailed study of the Matuyama-Brunhes polarity reversal (Leonhardt and Fabian, 2007) and Laschamp excursion (Leonhardt et al., 2009) the field instability starts when reverse flux patches appear in low or mid latitude regions at the CMB and then move poleward. In contrast, Aubert et al. (2008) found a mixed behavior, with reversals and excursions initiated by reversed flux generated both outside and inside the tangent cylinder. The same authors suggested that the appearance of the South Atlantic reversed flux patch could be attributed to a reverse magnetic anticyclone supplied by a strong equatorial magnetic upwelling.

The most recent geomagnetic dipole field is decreasing very rapidly and its temporal linear extrapolation would predict a null field at around $1000 \mathrm{yr}$ from now. In some parts of the Earth's surface this zero value would be reached even earlier since this field is more complex than a pure dipolar field: for instance, in the polar regions the field would be zero in around $300 \mathrm{yr}$ (De Santis, 2007). Some other papers (De Santis et al., 2004; De Santis and Qamili, 2008, 2010a) have found clear evidence for a chaotic state of the present geomagnetic field. De Santis $(2007,2008)$ calculated the Shannon information, which is a measure of the spatial order, for the field of the last $400 \mathrm{yr}$. He found that the Shannon information started to decay from around 1690, and began to decrease more rapidly at around 1775 and even more rapidly after 1900, revealing that the field is increasing its overall complexity. The author also found that some parts of the globe (e.g., Antarctica) contribute more than others to this trend, in agreement with what was found by Gubbins (1987).

All these aspects can be interpreted as a sign that the Earth's magnetic field might be in the early stage of a reversal (Hulot et al., 2002; De Santis et al., 2004; but see also Constable and Korte, 2006). Other authors, studying the future evolution of the field from numerical dynamos, use more caution in interpreting these results (Hulot et al., 2010). Analyzing the exponential growth of errors in numerical models, these authors concluded that predictions for the next reversal will not be possible for more than one century, although better predictions for the evolution of the field in the near future could be possibly made.

Recently, De Santis and Qamili (2010b) focused their attention on the South Atlantic Anomaly (SAA), which is a great depression of the geomagnetic field at the Earth's surface. These authors proposed a simple model to represent the dynamics of this feature in terms of an apparent monopolar magnetic source moving at the top of the outer core. In practice, under the frozen flux approximation in the outer core (strong coupling between material motions and magnetic fields), this would represent the magnetic expression of a vortex in the outer core, as a component of a strong magnetic flux with reversed polarities with respect to the surroundings. The origin of the SAA can be either due to a decrease in the whole geomagnetic field strength or in the dipole field, which are two typical ingredients for a possible geomagnetic reversal. Another cause could be an increase in the field complexity, i.e., an increase (decrease) in the corresponding Shannon entropy (information), which has been recently revealed as another important ingredient for a possible polarity change (De Santis et al., 2004; De Santis and Qamili, 2008). Therefore, we could postulate that a possible imminent reversal would be preceded by a significant increase in the reversed magnetic flux in the CMB, and in turn at the Earth's surface, in the SAA area. In this paper we study the surface extension of this anomaly over the last $400 \mathrm{yr}$. In particular we analyze the variation in space and time of the area included by the $32000 \mathrm{nT}$ isoline as deduced from GUFM1 (1590-1990; Jackson et al., 2000) and IGRF-11 (1900-2010; Finlay et al., 2010) global models of the geomagnetic field. The combined time series was obtained with a point every $5 \mathrm{yr}$ taking the values from GUFM1 in the period 1590-1955, and IGRF-11 afterward (at 1960 the two models agree quite well). Then we will fit it with a nonlinear function usually characterizing a system under a significant change of state, the so-called "critical" or "tipping point". The $32000 \mathrm{nT}$ isoline was chosen as a reference because it is the lowest value in the time interval of study, so it is easy to follow the increase in the SAA extent with time. In the GUFM1 model this isoline appears at the beginning of the interval of the model validity; some recent papers (Gubbins et al., 2006; Finlay, 2008) have cast some doubts on the validity of the back linear extrapolation in time of the $g_{1}^{0}$ coefficient (related to the axial dipole of the field) before 1840 , which is just after the time Gauss introduced an absolute method to measure the geomagnetic field intensity in 1832 (Malin, 1982). In the following we will denote with GUFM1-G and GUFM1-F the two models derived from the suggestions given by Gubbins et al. (2006) and Finlay (2008), respectively, whose models differ from GUFM1 mainly in the values of $g_{1}^{0}$ before 1840 (the other Gauss coefficients are just rescaled appropriately). 
In the next section we will introduce some concepts related to critical point processes, i.e., dynamical systems coping with dramatic changes of state, and then we will apply these concepts to the temporal evolution of SAA area extension over the last $400 \mathrm{yr}$, together with the changes in the mean global sea level (GSL) as provided by Jevrejeva et al. (2008) and Church and White (2011) (but for an alternative view on GSL please see Mörner, 2004, 2010). The comparison between SAA and GSL is important because an unexpectedly close correlation between these quantities has recently been found (De Santis et al., 2012). Our joint analysis will confirm the existence of a tipping point for both time series. Finally we will present some conclusions and discussions.

\section{Critical point processes and critical time}

Many complex systems have "critical" thresholds (the socalled critical or tipping points) at which the system moves abruptly from one state to another, i.e., shifts toward a critical transition (Scheffer, 2009); the corresponding times are also called critical times. In the literature we can find different methods for scientific predictions of catastrophic events based on the concepts of non-linear physics (e.g., Bunde et al., 2002; Dakos et al., 2012). A way to attempt to recognise these critical transitions is to detect some early warnings that may anticipate them (Scheffer et al., 2009). This strategy has been applied in ecology, medicine and global finance (May et al., 2008). Another approach is related to the critical point hypothesis for processes usually characterized by some cumulative critical quantity. This approach has also found applications in such different fields as: climate dynamics, seismology, material rupture, financial crashes, etc. (Sornette, 2003). It is important to note that the critical point hypothesis can be used when the system is close to or moving toward a critical state, in analogy with a phase transition (e.g., Stanley, 1971), and the capability to predict the critical point generally improves as the latter is more approaching. With the term "critical" we denote the state of a system between order and disorder, and which is strongly influenced by external and internal factors. Examples of systems that respond to such characteristics are some cases of liquids and magnets, but many others can be found in different disciplines (Sornette, 2006; Scheffer et al., 2012).

In analogy with standard critical phenomena of solid state physics, it is thought that the precursory seismicity of large events may follow power laws or alternative diverging functions in time. This approach has found more applications in the attempt to predict large earthquakes, although mostly from a retrospective point of view. In particular, Bufe and Varnes (1993) and Bowman et al. (1998) suggested that the time $t_{\mathrm{c}}$ of the largest main shock of a seismic sequence is the critical time of the seismic sequence, i.e., the time when the system drastically changes its dynamical regime. Since the seismological phenomena are mainly earthquakes, which are large ruptures or failures of a part of the crust, this approach has also been called the "time-to-failure" approach. In a broad sense, also in other occasions and fields when a general system shifts to a critical transition, the latter event could be considered as a failure of the system to maintain its previous typical state; thus the term failure must be taken in this general meaning, not implying necessarily that there is a physical failure or rupture in the system under study. Then, a measure $y(t)$ of the seismic release (e.g., the seismic deformation) at any preceding time $t$ reasonably close to the time $t_{\mathrm{c}}$ can be described by a power law relation of the form:

$y(t)=k\left(t_{\mathrm{c}}-t\right)^{-n}$

where $k>0$ and $1>n>0$ are appropriate parameters. Equation (1) is characterized to have a singularity at $t=t_{\mathrm{c}}$ because $y\left(t_{c}\right)=\infty$. In practice, in seismology it is preferred to integrate Eq. (1) in time to use a cumulative function $s(t)$ of $y(t)$, in order to have a finite value for $s\left(t_{\mathrm{c}}\right)$, its time derivative being singular, i.e., the slope of the function $s(t)$ at $t_{\mathrm{c}}$ is vertical. In this way, we have:

$s(t)=\int y(t) \mathrm{d} t=a-\frac{k}{m}\left(t_{\mathrm{c}}-t\right)^{m}=a+b\left(t_{\mathrm{c}}-t\right)^{m}$

where $a>0$ is the constant of integration; $b=-k / m<0$, and $m=1-n>0$ are constant parameters that are found by means of a nonlinear least regression on the available data; $m$, normally $0.2<m<0.6$ (Mignan, 2011), is a critical exponent that represents the degree of accelerating energy release (De Santis et al., 2010). It is clear that $a$ is the value of the measure related to the cumulative seismic release at the critical time, i.e., $a=s\left(t_{\mathrm{c}}\right)$. In addition to the accelerating strain release in Eqs. (1) or (2), Sornette and Sammis (1995) proposed an extension of this method, finding a better fit to the time of occurrence of large seismic events by fitting a function that included a log-periodic fluctuation:

$s(t)=a+b\left(t_{\mathrm{c}}-t\right)^{m} \cdot\left\{1+d \cdot \cos \left[2 \pi f \ln \left(t_{\mathrm{c}}-t\right)+\varphi\right]\right\}$

where $d$ is the magnitude of the fluctuations around the acceleration growth, $f$ is the frequency of the fluctuations, $\varphi$ is the phase shift, and $t_{\mathrm{c}}$ is the critical time. Note that for $d=0$ we have the simple power law as in Eq. (2). The equations from Eqs. (1) to (3) have also been applied in analyzing financial crises (Sornette, 2003).

An alternative form of diverging functions in time is that of considering just a logarithmic function in (reversed) time (e.g., Vandewalle et al., 1998):

$s(t)=A+B \ln \left(t_{\mathrm{c}}-t\right)$

where $A>0$ and $B<0$ (and $t_{\mathrm{c}}$ ) are parameters to be found from the experimental data, thus reducing the unknown parameters from four of Eq. (2) to only three. With respect to Eq. (2), but as for Eq. (1), the price to pay of Eq. (4) is that we have $s\left(t_{\mathrm{c}}\right)=\infty$ at $t=t_{\mathrm{c}}$ and $A=s\left(t_{\mathrm{c}}-1\right)$. Since in our 


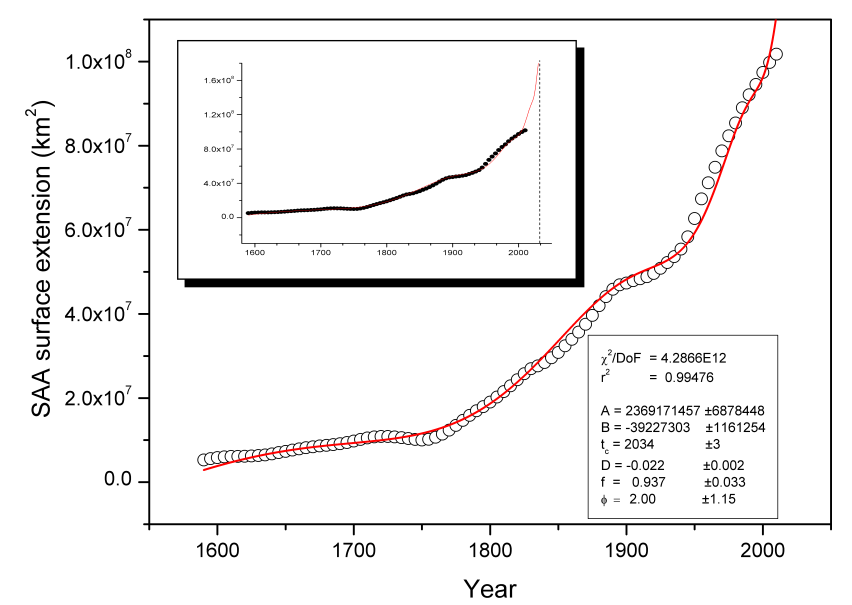

Fig. 1. Extension of the SAA over the last $400 \mathrm{yr}$ and the best nonlinear fit of the function indicated in the text as Eq. (5). The "critical time" $t_{\mathrm{c}}$ would be $2034 \pm 3 \mathrm{yr}$, where the curve will have a singularity, i.e., where the curve is tangent to the vertical dashed line drawn at the critical time in the smaller picture. Our interpretation is that this time will represent the time of no return for a great change in the geomagnetic field, possibly going toward a reversal or excursion. In the inset table, DoF are the degrees of freedom and $r$ is the correlation coefficient of the nonlinear fit; for the other fitting parameters see the text.

calculations the time is in years, the value of $A$ is a good approximation of the actual value that the quantity under study will take close to its critical time (i.e., just one year before). Equation (4) is the time integral of the limiting case of Eq. (1) with $n=1$, and $A$ is the constant term of integration. The corresponding log-periodic form can be written as (e.g., Vandewalle et al., 1998):

$s(t)=A+B \ln \left(t_{\mathrm{c}}-t\right) \cdot\left\{1+D \cdot \cos \left[2 \pi f \ln \left(t_{\mathrm{c}}-t\right)+\varphi\right]\right\}$.

Note that for $D=0$ we have simple logarithmic divergence as in Eq. (4).

It is clear that the "integral" Eqs. (2)-(5) are more appropriate than Eq. (1) for SAA and GSL, because they are all cumulative processes as the seismic deformation for which some of those equations had been introduced.

The quality of the acceleration toward the critical point can be evaluated by the $C$ factor (Bowman et al., 1998), which measures the ratio between the root mean squares (rms) of the diverging function $\left(\mathrm{rms}_{\mathrm{df}}\right)$ and the rms of the best fit line $\left(\mathrm{rms}_{\text {line }}\right)$ :

$C=\frac{\mathrm{rms}_{\mathrm{df}}}{\mathrm{rms}_{\text {line }}}=\sqrt{\frac{1-r_{\mathrm{df}}^{2}}{1-r_{\text {line }}^{2}}}$

where $r$ is the correlation coefficient of the corresponding fit. The lower than 1 the $C$ factor is, the greater (and more significant) the acceleration toward the critical point is.

In the next section we will analyze the SAA at the Earth's surface, because the geomagnetic field is known there and

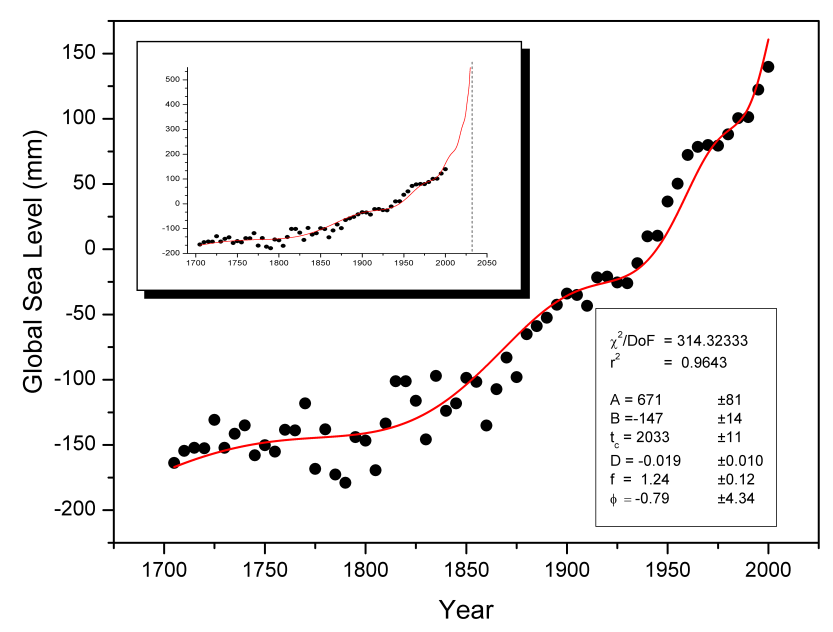

Fig. 2. Global sea level (GSL) rise and its best log-periodic fit with Eq. (5). The critical time $(2033 \pm 11 \mathrm{yr}$ indicated by the vertical dashed line in the smaller picture) within the given error is the same as that estimated for the SAA. In the inset table, DoF are the degrees of freedom and $r$ is the correlation coefficient of the nonlinear fit; for the other fitting parameters see the text.

any global model is more reliable at the Earth's surface than at that extrapolated at the $\mathrm{CMB}$, where the main sources of the geomagnetic field are placed (e.g., Merrill and McElhinny, 1983): the higher harmonics, which are typically measured at the surface with a low signal-to-noise ratio, are greatly amplified together with their errors, when extrapolated downward to the $\mathrm{CMB}$, contaminating any final representation of the field at that depth (Lowes, 1974). In the Appendix we show that the critical time $t_{\mathrm{c}}$ for the SAA is as important at the Earth's surface as at the CMB.

\section{Application to SAA and GSL data and interpretation: a great planetary change?}

We applied all possible functions given by Eqs. (1)-(5) over the SAA and GSL data. In our study the log-periodic approach Eq. (5) has shown the best fit over the available data with respect to the other possible functions in terms of the lowest $\chi^{2}$ and the highest correlation coefficient $r$. Figures 1 and 2 show the corresponding results for SAA and GSL, respectively. A low $C$ factor ( 0.18 and 0.48 for SAA and GSL, respectively) confirms a significant acceleration toward the critical point. When we compare the couples of the same fitting parameters with each other, the agreement is astonishing for most of them: in particular, the critical time $t_{\mathrm{c}}$ is practically the same (around $2034 \pm 3 \mathrm{yr}$ and $2033 \pm 11 \mathrm{yr}$, for SAA and GSL, respectively; please note that the indicated errors are only statistical because they could be up to two times greater, Gross and Rundle, 1998); when the fit is applied to GUFM1-G and GUFM1-F the results change a little, with a critical time ranging from 2014 to 2027 . In the above 
analyses we did not consider any error in the SAA area estimates. Defining an accurate error budget for the area of the SAA is not possible.

Not only has one to find what the accuracy of the Gauss coefficients is, but one also has to estimate what the contributions of the unknown small scales of the magnetic field are. One also has to estimate what effect the regularization process (if present) applied for deriving magnetic field models from geomagnetic data has on the SAA area. Nevertheless we expect that the greatest contribution will come from the Gauss coefficient errors, so we try to take them into account in a simple way. Likely, errors in the Gauss coefficients change with time, say from $10 \%$ at the beginning of the considered time interval and $1 \%$ in more recent times, so we cannot be too wrong in supposing an average crude error budget of $5 \%$ to propagate with the same percentage to the SAA area values. When these errors are considered in a weighted log-periodic fit the results (not shown here) are not significantly different from those above (in particular, we find a critical time of 2042). Therefore, in all cases a critical process is still compatible with model data. This means that the overall trend that underlies both quantities (SAA and GSL) is something real and not an artefact. This confirms the choice of De Santis et al. (2012) to make the comparison of SAA and GSL (in terms of Spearman rank correlation and relative entropy) without removing any trend (although, when removing a trend and normalizing both time series to unitary standard deviation, correlation still remains significant, with the Pearson correlation coefficient $r=0.62$ and $P<0.0001$; this correlation increases much more when we consider more recent data after 1800, reaching $r=0.94$ and $P<0.0001$ ). The low values of $\chi^{2} / \mathrm{DoF}$ (degrees of freedom) and the high values of the correlation coefficient $r$ (for both quantities $r>0.98$ ), with respect to the corresponding fit, indicate that the acceleration of both SAA and GSL is unlikely to be a mere coincidence, and that they are, rather, indications of some physical underlying critical point process. Also, the $D$ and $f$ parameters are very similar in both SAA and GSL, indicating that the fluctuations affect the acceleration in almost the same way in both physical quantities. In addition, it is interesting to note that the critical time of the SAA will be almost the time at which the SAA area, i.e., the parameter $A$, will cover a hemisphere: because of the validity of Eqs. (A2) and (A3), this is limited not only to the field at the Earth's surface, but would also be at the CMB, where $A^{\prime}$ of Eq. (A4) will cover more than half of the core surface. Since the SAA is usually considered the manifestation at the Earth's surface of a reversal magnetic flux produced at the CMB (e.g., Hulot et al., 2002), the epoch when the SAA may reach the area corresponding to the surface of half the planet is a critical moment for the present geomagnetic field. This time is not the time of the eventual geomagnetic reversal, but we interpret it as the time of the point of no return, after which the geomagnetic field could fall in the process of a global geomagnetic transition, which could be a rever- sal or excursion of polarities. How long after the critical time $t_{\mathrm{c}}$ this transition will occur cannot be fully established, because what we predict is a time when the dynamical system reaches its critical state, after which any successive time is a potential candidate for the actual start of the reversal or excursion. Why GSL also shows the same overall trend with similar parameters is a question that deserves further scrutiny and is left to future work. What we can speculate now is that when GSL reaches its critical point it will correspond to a significant coverage of many present coasts, implying a big change in the land-ocean system. In addition, the similarities found in both SAA and GLS confirm that the two quantities are really closely related, and, if the interpretation of an imminent geomagnetic field reversal is correct, this would once more support the internal hypothesis indicated among other possibilities in De Santis et al. (2012).

\section{Conclusions}

In this work we analyze both SAA and GSL overall trends in the last few centuries, finding an astonishing similarity, further confirming previous results (De Santis et al., 2012). These similar trends can be explained by the theory of the critical point processes for which each dynamical system is close to or is going toward a critical point, when the system will undergo a dramatic change in its macroscopic properties. This interpretation comes from the analysis of the SAA behavior, for which the critical time $t_{\mathrm{c}}$ would correspond to practically the time at which the SAA area will exceed the extent of a hemisphere. Since SAA is a superficial manifestation of a reverse magnetic flux at the CMB, this time will be the time of no return after which the geomagnetic field will go to a significant transition reverse in polarity, such as a geomagnetic excursion or a complete geomagnetic reversal.

A similar dramatic change would have to occur in the oceans, although no clear information can be obtained from the present work. Regarding this, only some questions can be asked: would the entire Earth or most of it be flooded? This seems not to be the case, because from a simple calculation (Woo, 2011), the predicted sea level rise of around $0.5 \mathrm{~m}$ higher than the present value (at time $t_{\mathrm{c}}-1$ ) will cause about $3 \mathrm{~km}$ of present coasts to be covered by water. Nevertheless, if this is the case, the consequences will be very dramatic as well (let us think of the many cities and mega-cities that are close to the coasts). Or would the GSL suddenly collapse? Or would the GSL's abrupt increase imply an enormous change in the land-ocean system? Or what else? In this sense, if the model we propose for both SAA and GSL is correct, what is in preparation will be a really global change, and many more parts of the planet could be involved, humankind included.

Of course, this interpretation must be taken with some caution at least for three reasons, so that it will need further investigation in the coming years. First, the SAA surface in the most recent years seems to deviate slightly from the overall 
fit, although this could simply be due to some edge effects in the IGRF-11 model construction that we used for the most recent years. Second, because of its intrinsic chaotic characteristics (De Santis et al., 2004), the time of predictability of the geomagnetic field is comparable with the remaining time to the predicted $t_{\mathrm{c}}$ (e.g., De Santis et al., 2004; Hulot et al., 2010). Thus, the prediction of the critical time should be updated again as soon as more SAA and GSL data become available, since any prediction based on a log-periodic function such as Eq. (5) is not stable when we are far from the critical time, but improves its quality of prediction as soon as we are closer to $t_{\mathrm{c}}$ (e.g., Brée and Joseph, 2013). The study of the diverging function parameters at successive predictions/times together with the use of the $C$ factor will also allow one to investigate any deviation of the real behavior from the prediction, and possibly to detect a change from the present "catastrophic" trend: any departure from the behavior predicted so far would be seen in terms of significant increase of both $t_{\mathrm{c}}$ and the $C$ factor. Third, both SAA and GSL can also be well fitted by some higher degree polynomial: for example, a quintic polynomial (containing the same number of unknown coefficients of our log-periodic function) provides, in terms of $r^{2}$ and $\chi^{2} / \mathrm{DoF}$, a fitting quality similar to that obtained by the log-periodic function, although, of course, the found polynomial behaves unrealistically outside the data range, thereby excluding its use for forecasting purposes.

Now one might ask why we are able to predict the point of no return from just a few hundred years of a phenomenon that usually lasts several thousand years (Jacobs, 1994; but see also Nowaczyk et al., 2012, where the Laschamp excursion seems to change the geomagnetic polarity in a few hundred years), i.e., with some analysis based on data taken over a temporal window much shorter than the typical timescales of the reversal or excursion process. A simple answer is that we are analyzing a sufficient (although short) time before the eventual critical transition: we believe that the recent acceleration of both SAA and GSL is nothing casual, but probably uncovers important physical information regarding the future of our planet in the near future, such as a possible precursor to the eventual close critical transition of the geomagnetic field.

\section{Appendix A}

This appendix has the aim of showing that the results obtained by means of analyses made on the SAA at the Earth's surface are equivalent to those made at the CMB, where the main sources of the geomagnetic field are placed, but where any extrapolation is difficult or even impossible.

However complicated the geomagnetic field may be at the SAA within the $32000 \mathrm{nT}$ isoline, we can define a frustum of quasi-cone $\Omega$ that is confined by the lower surface $S\left(r_{\mathrm{CMB}}\right)$ at the $\mathrm{CMB}$, i.e., at $r=r_{\mathrm{CMB}}=3485 \mathrm{~km}$ and the upper surface $S\left(r_{0}\right)$ of the SAA at the Earth's surface, i.e., at

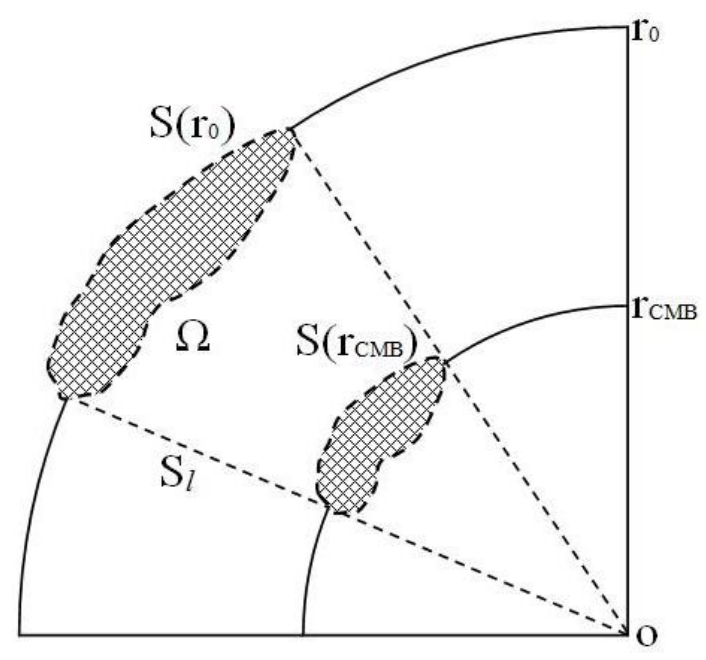

Fig. A1. The magnetic flux crossing both the core mantle boundary (CMB) and South Atlantic Anomaly (SAA) is conserved. Thus analyses on the SAA at the Earth surface are equivalent to those made at the CMB.

$r=r_{0}=6371 \mathrm{~km}$ (Fig. A1); the lateral surface is here called $S_{l}$. The lower surface $S\left(r_{\mathrm{CMB}}\right)$ at the $\mathrm{CMB}$ is representative of some typical isoline enclosing the reverse magnetic flux (we will come back to this in the final part of the Appendix).

The divergence-free condition of the geomagnetic field imposes a null flux through the surfaces bounding the volume $\Omega$ :

$\Phi\left[S\left(r_{\mathrm{CMB}}\right)\right]-\Phi\left[S\left(r_{0}\right)\right]-\Phi\left[S_{l}\right]=0$

where $\Phi\left[S_{i}\right]$ is the magnetic flux across the surface $S_{i}$ (where $S_{i}$ is $S\left(r_{0}\right)$ or $S\left(r_{\mathrm{CMB}}\right)$ or $\left.S_{l}\right)$ that can be expressed as follows:

$\Phi\left[S_{i}\right]=\int \boldsymbol{B}_{i} \cdot \boldsymbol{n} \mathrm{d} S_{i}=\overline{\boldsymbol{B}_{i} \cos \delta} S_{i}$

where $\overline{\boldsymbol{B}_{i} \cos \delta}$ is the mean component of the field perpendicular to the surface $S_{i}$ ( $\delta$ is the angle between the vector field $\boldsymbol{B}$ and the vector $\boldsymbol{n}$ normal to $S_{i}$ ). For the geometry of the quasi-conical volume, $\overline{\boldsymbol{B}_{i} \cos \delta}$ will be $\bar{Z}$ for the upper SAA and the lower surface at the CMB, while it will be a component in the horizontal plane for the lateral surface $S_{l}$. We can safely neglect the flux across the lateral surface $S_{l}$ of $\Omega$; thus Eq. (A1) becomes:

$\overline{Z\left(r_{\mathrm{CMB}}\right)} S\left(r_{\mathrm{CMB}}\right)=\overline{Z\left(r_{0}\right)} S\left(r_{0}\right)$

and

$S\left(r_{\mathrm{CMB}}\right)=\frac{\overline{Z\left(r_{0}\right)}}{\overline{Z\left(r_{\mathrm{CMB}}\right)}} S\left(r_{0}\right)=\gamma S\left(r_{0}\right)$,

where the $\gamma$ ratio

$\gamma=\overline{Z\left(r_{0}\right)} / \overline{Z\left(r_{\mathrm{CMB}}\right)}=\overline{S\left(r_{\mathrm{CMB}}\right)} / \overline{S\left(r_{0}\right)}$ 


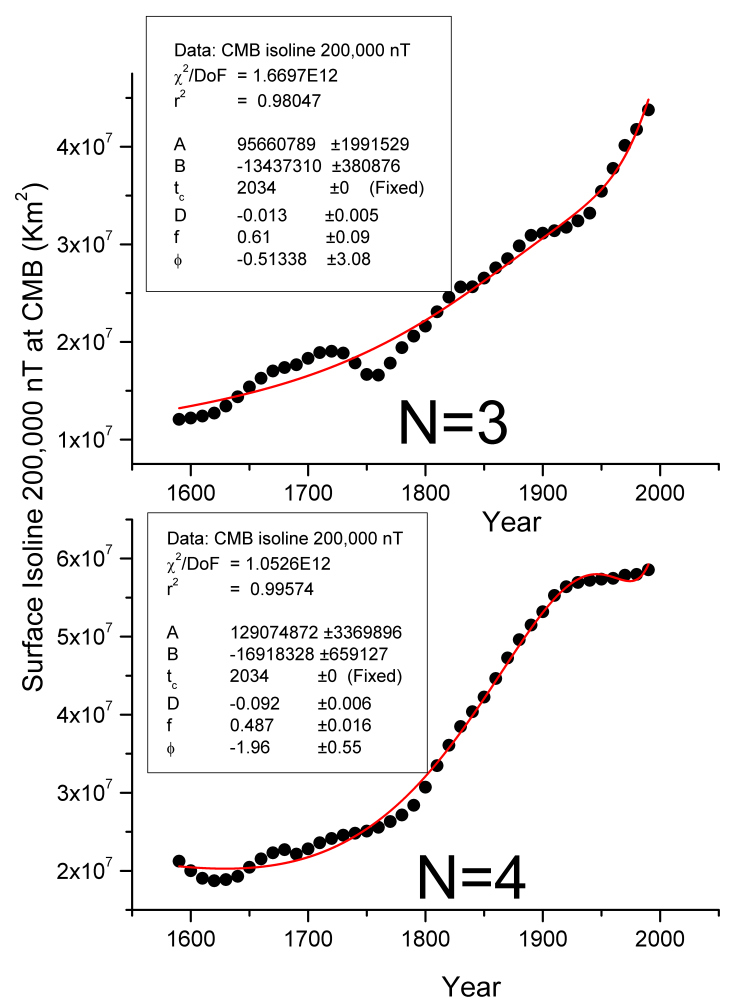

Fig. A2. Surface enclosed by the isoline $200000 \mathrm{nT}$ at the CMB for an expansion of GUFM1 up to the spherical harmonic degree $N=3$ and $N=4$. Both trends are almost monotonic and diverging in time. A log-periodic function with critical (a priori fixed) time of $2034 \mathrm{yr}$ is a reasonable fit for both cases.

can be taken as constant in time. This means that an equation of the same form as Eq. (4) (but this would also be valid for Eq. 5) can also be written for $s^{\prime}(t)$ of $S\left(r_{\mathrm{CMB}}\right)$ :

$s^{\prime}(t)=A^{\prime}+B^{\prime} \ln \left(t_{\mathrm{c}}-t\right)$,

with $A^{\prime}=\gamma A$ and $B^{\prime}=\gamma B$. Thus, the results we find at the Earth's surface are also representative of the deep dynamics of the geomagnetic field; in particular, the critical time $t_{\mathrm{c}}$ estimated at the Earth's surface will also be the same for the CMB.

Unfortunately it is difficult to verify the constancy of $\gamma$ with any global model (such as GUFM1) that is based on observational data taken at the Earth's surface. This difficulty is twofold: (i) the area $S\left(r_{\mathrm{CMB}}\right)$ is impossible to determine, and (ii) it is difficult or even impossible to estimate $\overline{Z\left(r_{\mathrm{CMB}}\right)}$ because of the eventual explosion of errors when continuing the vertical component from the Earth's surface to the CMB, because of their multiplication by a factor $\left[r_{0} / r_{\mathrm{CMB}}\right]^{n+2}(n$ is here the spherical harmonic degree of the geomagnetic field expansion).

To reasonably circumvent most of the problems, we can simply look at an isoline at the CMB that could act as the $32000 \mathrm{nT}$ at the Earth's surface. By applying a simple dipolar downward continuation of the $32000 \mathrm{nT}$ isoline to $\mathrm{CMB}$ (just multiplying by $\left[r_{0} / r_{\mathrm{CMB}}\right]^{3}$ ) we obtain about $200000 \mathrm{nT}$. Therefore, looking at the surface enclosed by the latter isoline at the CMB for an expansion of the GUFM1 model up to $N=3$ and $N=4$ (Fig. A2), we notice an almost monotonic trend for both cases where a log-periodic behavior pointing to a critical (a priori fixed) time of 2034 is something really possible ( $C$ factor is always much less than 1 for both cases: 0.52 and 0.23 for $N=3$ and 4 , respectively). By the way, the area of the enclosed surfaces $1 \mathrm{yr}$ before the critical time for $N=3$ and $N=4$ are $64 \%$ and $84 \%$, respectively, so in both cases the surface of this isoline at the critical time will cover more than half of the entire core surface. We limit our analysis made at the CMB to $N=4$, because for larger values of $N$, the expected downward continuation errors would be too large to reliably detect the $200000 \mathrm{nT}$ (or any other) isoline.

Acknowledgements. Part of this work has been realised in the frame of the SAGA-4-EPR project co-funded by the Italian Foreign Office, the Istituto Nazionale di Geofisica e Vulcanologia (Italy) and Northeastern University of Shenyang (China). We thank G. Hulot, G. Balasis and P. Lurcock, whose constructive comments helped us to improve a preliminary version of this manuscript. We also thank two anonymous referees for their comments and suggestions.

Edited by: R. Lasaponara

Reviewed by: two anonymous referees

\section{References}

Amit, H., Leonhardt, R., and Wicht, J.: Polarity reversals from paleomagnetic observations and numerical dynamo simulations, Space Sci. Rev., 155, 293-335, 2010.

Aubert, J., Aurnou, J., and J. Wicht, J.: The magnetic structure of convection-driven numerical dynamos, Geophys. J. Int., 172, 945-956, 2008.

Bowman, D. D., Ouillon, G., Sammis, C. G., Sornette, A., and Sornette, D.: An observational test of the critical earthquake concept, J. Geophys. Res., 103, 24359-24372, 1998.

Brée, D. S. and Joseph, N. L.: Testing for financial crashes using the $\log$ periodic power law model, International Review Financial Analysis, 30, 287-297, 2013.

Bufe, C. G. and Varnes, D. J.: Predictive modelling of the seismic cycle of the Greater San Francisco Bay region, J. Geophys. Res., 98, 9871-9883, 1993.

Bunde, A., Kropp, J., and Schellnhuber, H. J.: The Science of Disasters. Climate disruptions, heart attacks, and market crashes, Springer Berlin, 2002.

Cande, S. C. and Ken, D. V.: Revised calibration of the geomagnetic polarity timescale for the late Cretaceous and Cenozoic, J. Geophys. Res., 100, 6093-6095, 1995.

Christensen, U. R.: Geodynamo models: Tools for understanding properties of the Earth's magnetic field, Phys. Earth Planet. Int., 187, 157-169, 2011.

Church, J. A. and White, N. J.: Sea-level rise from the late 19th to the early 21 st century, Surv. Geophys., 32, 585-602, 2011. 
Constable, C. G.: Modelling the geomagnetic field from syntheses of paleomagnetic data, Phys. Earth Planet. Int., 187, 109-117, 2011.

Constable, C. G. and Korte, M.: Is Earth's magnetic field reversing?, Earth Planet. Sci. Lett., 246, 1-16, 2006.

Courtillot V. and Besse J.: Magnetic Field Reversals, Polar Wander, and Core-Mantle Coupling, Science, 237, 1140-1145, 1987.

Dakos, V., Carpenter, S. R., Brock, W. A., Ellison, A. M., Guttal, V., Ives, A. R., Kéfi, S., Livina, V., Seekell, D. A., van Nes, E. H., and Scheffer, M.: Methods for detecting early warnings of critical transitions in time series illustrated using simulated ecological data, PLoS One, 7, e41010, doi:10.1371/journal.pone.0041010, 2012.

De Santis, A.: How persistent is the present trend of the geomagnetic field to decay and, possibly, to reverse?, Phys. Earth Planet. Int., 162, 217-226, 2007.

De Santis, A.: Erratum to "How persistent is the present trend of the geomagnetic field to decay and, possibly, to reverse?", Phys. Earth Plan. Int., 170, p. 149, 2008.

De Santis, A. and Qamili, E.: Are we going towards a global planetary magnetic change? 1st WSEAS International Conference on Environmental and Geological Science and Engineering (EG'08), 149-152, 2008.

De Santis, A. and Qamili, E.: Shannon information of the geomagnetic field of the past 7000 years, Nonlin. Proc. Geophys., 17, 77-84, 2010a.

De Santis, A. and Qamili, E.: Equivalent monopole source of the geomagnetic South Atlantic Anomaly, Pure Appl. Geophys., 167, 339-347, 2010b.

De Santis, A., Tozzi, R., and Gaya-Piqué, L.R.: Information content and K-Entropy of the present geomagnetic field, Earth Planet. Sci. Lett., 218, 269-275, 2004.

De Santis, A., Cianchini, G., Qamili, E., and Frepoli, A.: The 2009 L'Aquila (Central Italy) seismic sequence as a chaotic process, Tectonophysics, 496, 44-52, 2010.

De Santis, A., Qamili, E., Spada, G., and Gasperini, P.: Geomagnetic South Atlantic Anomaly and global sea level rise: a direct connection?, J. Atmos. Sol. Terr. Phys., 74, 129-135, 2012.

Finlay, C. C.: Historical variation of the geomagnetic axial dipole, Phys. Earth Planet. Int., 170, 1-14, 2008.

Finlay, C. C., Maus, S., Beggan, C. D., Hamoudi, M., Lowes, F. J., Olsen, N., and Thebault, E.: Evaluation of candidate geomagnetic field models for IGRF-11, Earth Planets Space, 62, 787$804,2010$.

Glatzmaier, G. A. and Roberts, P. H.: A three-dimensional selfconsistent computer simulation of a geomagnetic field reversal, Nature, 377, 203-209, 1995.

Gross, S. and Rundle, J.: A systematic test of time-to-failure analysis, Geophys. J. Int., 133, 57-64, 1998.

Gubbins, D.: Mechanism for geomagnetic polarity reversals, Nature, 326, 167-169, 1987.

Gubbins, D., Jones, A. L., and Finlay, C. C.: Fall in Earth's Magnetic Field is erratic, Science, 312, 900-902, 2006.

Hulot, G., Eymin, C., Langlais, B., Mandea, M., and Olsen, N.: Small-scale structure of the geodynamo inferred from Øersted and Magsat satellite data, Nature, 416, 620-623, 2002.

Hulot, G., Lhuillier, F., and Aubert, J.: Earth's dynamo limit of predictability, Geophys. Res. Lett., 37, L06305, doi:10.1029/2009GL041869, 2010.
Jackson, A., Jonkers, A. R. T., and Walker, M. R.: Four centuries of geomagnetic secular variation from historical records, Philos. Trans. R. Soc. Lond. A, 358, 957-990, 2000.

Jacobs, J. A.: Reversals of the Earth's magnetic field, 2nd Edition, Cambridge University Press, Cambridge, UK, 346 pp., 1994.

Jevrejeva, S., Moore, J. C., Grinsted, A., and Woodworth, P. L.: Recent global sea level acceleration started over 200 years ago?, Geophys. Res. Lett., 35, L08715, doi:10.1029/2008GL033611, 2008.

Leonhardt, R. and Fabian, K.: Paleomagnetic reconstruction of the global geomagnetic field evolution during the Matuyama/Brunhes transition: iterative Bayesian inversion and independent verification, Earth Planet. Sci. Lett., 253, 172-195, 2007.

Leonhardt, R., Fabian, K. Winklhofer, M. Ferk, A. Kissel, C., and Laj, C.: Geomagnetic field evolution during the Laschamp excursion, Earth Planet. Sci. Lett., 278, 87-95, 2009.

Lowes, F. J.: Spatial power spectrum of the main geomagnetic field, and extrapolation to the core, Geoph. J. R. Astr. Soc., 36, 717730, 1974.

Malin, S. R. C.: Sesquicentenary of Gauss's first measurement of the absolute value of magnetic intensity, Philos. Trans. R. Soc. Lond. A, 306, 5-8, 1982.

May, R. M., Levin, S. A., and Sugihara, G.: Ecology for bankers, Nature, 451, 893-895, 2008.

Merrill, R. T. and McElhinny, M. W.: The Earth's Magnetic Field (Its History, Origin and Planetary Perspective), Academic Press, San Diego, 1983.

Mignan, A.: Retrospective on the Accelerating Seismic Release (ASR) hypothesis: controversy and new horizons, Tectonophysics, 505, 1-16, 2011.

Mörner, N.-A.: Estimating future sea level changes from past records, Global Planet. Change, 40, 49-54, 2004.

Mörner, N.-A.: Some problems in reconstruction of mean sea and its changes with time, Quatern. Int., 221, 3-8, 2010.

Nowaczyk, N. R., Arz, H. W., Frank, H. W., Kind, J., and Plessen, B.: Dynamics of the Laschamp geomagnetic excursion from Black Sea sediments, Earth Planet. Sci. Lett., 351, 54-69, 2012.

Olson, P. and Amit, H.: Changes in earth's dipole, Naturwissenschaften, 93, 519-542, 2006.

Olson, P., Driscoll, P., and Amit, H.: Dipole collapse and reversal precursors in a numerical dynamo, Phys. Earth Planet. Int., 173, 121-140, 2009.

Raup D. M.: Magnetic Reversals and Mass extinctions, Nature, 314, 341-343, 1985.

Scheffer, M.: Critical Transitions in Nature and Society. Princeton Univ. Press, 2009.

Scheffer, M., Bascompte, J., Brock, W., Brokvin, V., Carpenter, S. R., Dakos, V., Held, H., van Nes, E. H., Rietkerk, M., and Sugihara, G.: Early-warning signals for critical transitions, Nature, 461, 53-59, 2009.

Scheffer, M., Carpenter, S. R., Lenton, T. M., Bascompte, J., Brock, W., Dakos, V., van de Koppel, J., van de Leemput, I. A., Levin, S. A., van Nes, E. H., Pascual, M., and Vandermeer, J., Anticipating critical transitions, Science, 338, 344-348, 2012.

Sornette, D.: Why stock markets crash. Critical events in complex financial systems, Princeton Univ. Press, Oxford, 2003.

Sornette, D.: Critical Phenomena in Natural Sciences, Second Ed. Springer, Berlin, 2006. 
Sornette, D. and Sammis, C.: Complex critical exponents from renormalization group theory of earthquakes: implications for earthquake predictions, J. Phys. I France, 5, 607-619, 1995.

Stanley, H. E.: Phase transition and critical phenomena, Clarendon Press, New York, 1971.

Takahashi, F., Matsushima, M., and Honkura, Y.: Simulations of a quasi-Taylor state geomagnetic field including polarity reversals on the Earth simulator, Science, 309, 459-461, 2005.

Vandewalle, N., Ausolos, M., Boveraus, P., and Minguet, A.: How the financial crash of October 1997 could have been predicted, Eur. Phys. J. B., 4, 139-141, 1998.
Wicht, J. and Christensen, U. R.: Torsional oscillations in dynamo simulations, Geophys. J. Int., 181, 1367-1380, 2010.

Wicht, J. and Olson P.: A detailed study of the polarity reversal mechanism in a numerical dynamo model, Geochem. Geophys. Geosyst., 5, Q03H10, doi:10.1029/2003GC000602, 2004.

Wicht, J., Stellmach, S., and Harder, H.: Numerical models of the geodynamo: from fundamental Cartesian models to 3-D simulations of field reversals, edited by: Glassmeier, K. H., Soffel, H., and Negendank, J. F. W., Geomagnetic field variations. Springer, Berlin, 107-158, 2009.

Woo, G.: Calculating Catastrophe, Imperial College Press, 355 pp., 2011. 